\title{
MODELAGEM DA TRANSESTERIFICAÇÃO DO ÓLEO DE SOJA COM CATALISADOR HETEROGÊNEO CTA-MCM-41
}

\author{
J. P. BARBOSA ${ }^{2 *}$, F. A.COELHO ${ }^{1}$ e D. CARDOSO ${ }^{2}$ \\ ${ }^{1}$ Universidade Estadual de Campinas, Faculdade de Engenharia Química \\ ${ }^{2}$ Universidade Federal de São Carlos, Departamento de Engenharia Química \\ E-mail para contato: dilson@ufscar.br
}

\begin{abstract}
RESUMO - A reação de transesterificação é a rota utilizada atualmente para produção de biodiesel, via catálise homogênea. Entretanto, os catalisadores heterogêneos surgem como alternativa aos inconvenientes da rota atual, como a necessidade de etapas de purificação e neutralização. Um catalisador heterogêneo que tem se mostrado ativo em uma reação modelo que representa de forma satisfatória a reação de transesterificação de óleo vegetal é a CTA-MCM-41. Assim, este trabalho tem como objetivo a síntese do catalisador MCM-41 e a modelagem da sua aplicação na reação de transesterificação do óleo de soja. Para a determinação das constantes da cinética foi utilizada uma estratégia de simulação/otimização com algoritmo genético para ajustar o modelo aos dados experimentais obtidos através de um sistema reacional com reator em escala de bancada. Foi observado que o modelo proposto descreve bem os dados experimentais.
\end{abstract}

\section{INTRODUÇÃO}

O biodiesel foi introduzido na matriz energética nacional desde 2005 por meio do Programa Nacional de Produção e Uso de Biodiesel (PNPB) (COSTA, 2011). Este biocombustível tem excelentes propriedades como combustível motor, de modo que pode ser utilizado em motores de ignição por compressão a diesel. A sua utilização conduz a uma diminuição do dióxido de carbono, dióxido de enxofre, hidrocarbonetos não queimados e partículas geradas no processo de combustão (LIMA, 2005).

O processo que ainda hoje predomina para produção de biodiesel é a reação de transesterificação metílica via catálise homogênea básica, empregando como catalisadores o hidróxido de potássio $(\mathrm{KOH})$ ou o hidróxido de sódio $(\mathrm{NaOH})$. A transesterificação é uma importante classe de reações orgânicas na qual um éster é transformado em outro através da troca dos grupos alcóxidos. Na transesterificação de óleos vegetais, um triglicerídeo reage com um álcool na presença de um catalisador, produzindo uma mistura de ésteres monoalquídicos de ácidos graxos e glicerol, Figura 1. O processo global de transesterificação é uma sequência de três reações reversíveis e consecutivas, em que os monoglicerídeos e os diglicerídeos são os intermediários (BORGES e DÍAZ, 2012).

No entanto, a produção de biodiesel via catálise homogênea básica possui algumas desvantagens tais como a produção de sabões pela neutralização dos ácidos graxos livres ou pela saponificação de ésteres, bem como a dificuldade de purificação dos produtos formados. Visando contornar tais inconvenientes, novos

*Endereço atual: Universidade Estadual de Campinas, Faculdade de Engenharia Química 
catalisadores estão sendo testados (COSTA, 2011).

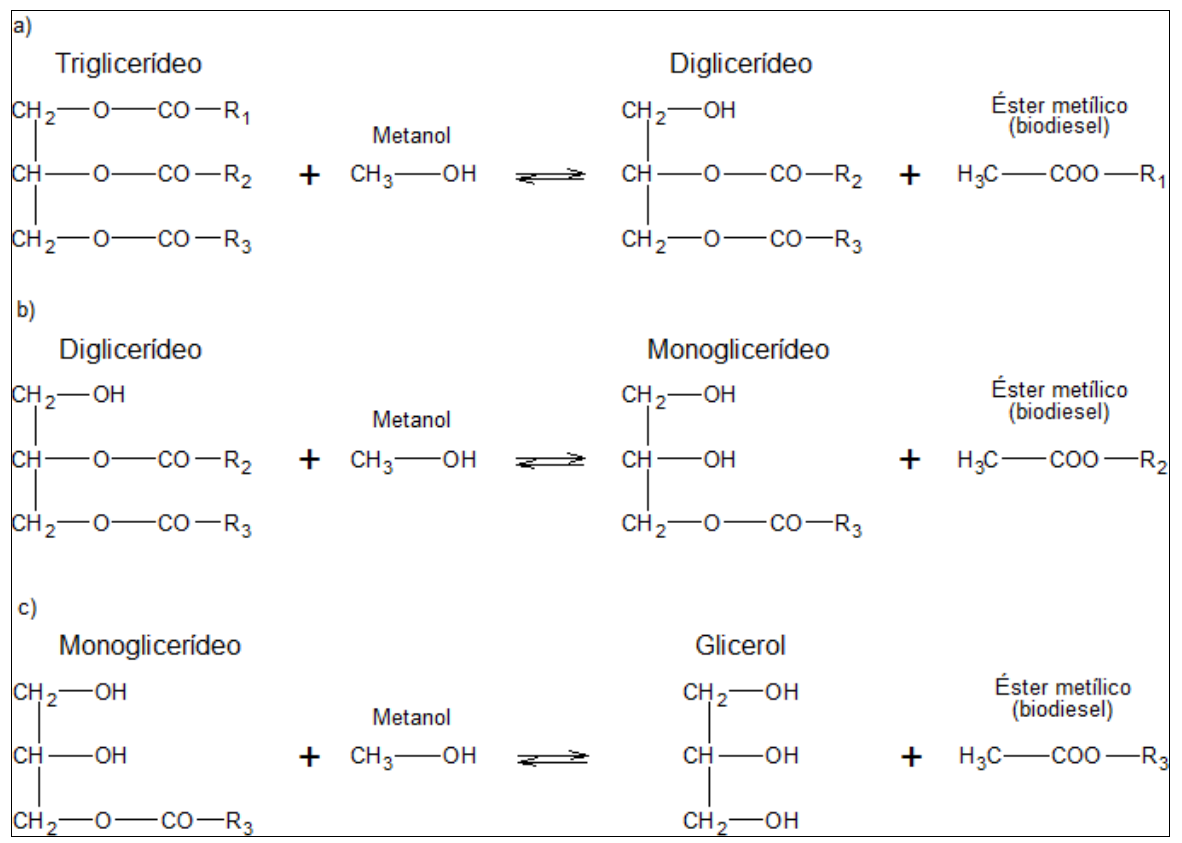

Figura 1 - Transesterificação de um triglicerídeo com um álcool.

O uso de catalisadores heterogêneos sanariam várias dessas dificuldades, já que é possível separá-los da mistura reacional sem o uso de solventes, sendo assim facilmente regenerado, o que possibilita o reaproveitamento desse material, apresentando também diminuição na corrosão dos equipamentos e contaminação dos produtos.

As peneiras moleculares microporosas e as mesoporosas com propriedades básicas apresentam potencial para aplicação em muitas reações de importância comercial como esta. Em particular, a peneira molecular mesoporosa MCM-41 que possui um sistema de poros ordenados de diâmetro ajustável entre 1,5 e $10 \mathrm{~nm}$ e área superficial muito alta - aproximadamente $1500 \mathrm{~m}^{2} / \mathrm{g}$ - vem sendo ajustada para possibilitar o seu uso como catalisador heterogêneo básico.

A CTA-MCM-41 tal como sintetizada, ou seja, com seus poros ocluídos com o cátion orgânico, foi aplicada como catalisador heterogêneo básico primeiramente por Kubota et al (2004) e em seguida por Martins e colaboradores (2006) na reação de Knoevenagel. Posteriormente Fabiano (2010) e Fabiano et al. (2010) estudaram a aplicação desse catalisador com propriedades básicas na transesterificação de monoésteres e de óleos vegetais e observaram resultados promissores, alcançando $78 \%$ de conversão no caso de monoésteres e $65 \%$ no caso do óleo vegetal.

Neste contexto, a modelagem da reação possibilitaria o dimensionamento e a otimização da operação de um reator catalítico utilizando a CTA-MCM-41. Assim, o presente trabalho tem por objetivo a síntese do catalisador CTA-MCM-41 com seus poros ocluídos com surfactante e o desenvolvimento de um modelo matemático para a sua aplicação na reação de transesterificação do óleo de soja. 


\section{PROCEDIMENTO EXPERIMENTAL}

A síntese do catalisador MCM-41 utilizado neste trabalho é baseada no método reportado por Mokhonoana et al (2010). O material mesoporoso foi sintetizado usando o trissilicato de sódio $\left(\mathrm{Na}_{2} \mathrm{O}\right.$ $\left.\cdot 3,45 \mathrm{SiO}_{2}\right)$, como fonte de sílica e o Brometo de cetiltrimetilamônio $\left(\mathrm{C}_{16} \mathrm{H}_{33} \mathrm{~N}\left(\mathrm{CH}_{3}\right)_{3} \mathrm{Br}-\mathrm{CTA}^{+} \mathrm{Br}\right)$ como surfactante orgânico e a composição molar do gel foi $3,45 \mathrm{SiO}_{2}: 1 \mathrm{Na}_{2} \mathrm{O}: 1,44 \mathrm{CTABr}: 350 \mathrm{H}_{2} \mathrm{O}$. Para o tratamento hidrotérmico foi utilizada a temperatura de $70^{\circ} \mathrm{C}$ e tempo de $48 \mathrm{~h}$. $\mathrm{O}$ catalisador foi caracterizado através da difratometria de raios $\mathrm{X}$ e termogravimetria.

O catalisador foi avaliado na reação de transesterificação com o óleo de soja utilizando metanol. A reação foi realizada em um reator Parr de bancada modelo 4590 (Figura 2). O controle de temperatura e pressão desse reator é feito com um controlador PID da Parr modelo 4848.

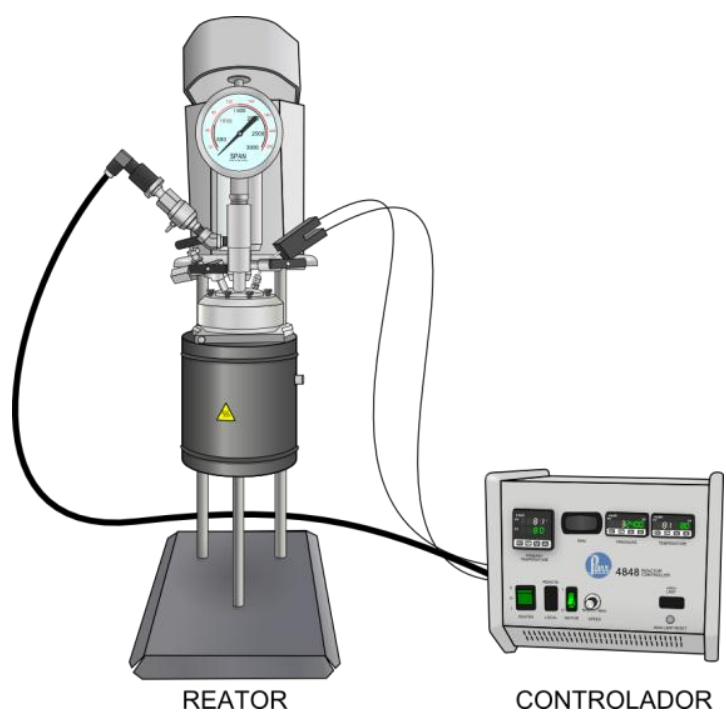

Figura 2 - Esquema do reator Parr e controlador usado na reação de transesterificação com óleo de soja.

As condições-padrão para a avaliação dos catalisadores foram: 5h de reação; temperatura constante de $80^{\circ} \mathrm{C}$; percentagem mássica de catalisador em relação ao óleo de $2 \%(1,25 \mathrm{~g})$ e razão molar óleo/metanol 1:6.

Os tempos para retirada das alíquotas foram: 15, 30, 45, 60, 120, 180, 240 e 300 minutos. As análises dessas alíquotas foram realizadas em um cromatógrafo da marca Varian, modelo ProStar 230 com detector de radiação ultravioleta e coluna de fase reversa C-18 modelo Shim-pack VP-ODS (4,6 mm x 25 cm e diâmetro de partícula de $5 \mu \mathrm{m})$.

\section{MODELO MATEMÁTICO}

A modelagem da cinética da transesterificação do óleo de soja foi feita baseada no modelo proposto por Noureddini e Zhu (1997), composto por um sistema de Equações Diferenciais Ordinárias (EDOs), apresentado nas Equações 1 a 6. Dado que o modelo originalmente foi proposto para reações homogêneas, uma modificação foi feita para levar em conta a massa de catalisador presente no meio reacional. 


$$
\begin{aligned}
& \frac{1}{m_{\text {cat }}} \frac{\mathrm{d} C_{\mathrm{T}}}{\mathrm{d} t}=-k_{1} C_{\mathrm{T}} C_{\mathrm{A}}+k_{2} C_{\mathrm{D}} C_{\mathrm{A}}-k_{7} C_{\mathrm{T}} C_{\mathrm{A}}^{3}+k_{8} C_{\mathrm{A}} C_{\mathrm{G}}^{3} \\
& \frac{1}{m_{\text {cat }}} \frac{\mathrm{d} C_{\mathrm{D}}}{\mathrm{d} t}=k_{1} C_{\mathrm{T}} C_{\mathrm{A}}-k_{2} C_{\mathrm{D}} C_{\mathrm{B}}-k_{3} C_{\mathrm{D}} C_{\mathrm{A}}+k_{4} C_{\mathrm{M}} C_{\mathrm{B}}^{3} \\
& \frac{1}{m_{\text {cat }}} \frac{\mathrm{d} C_{\mathrm{M}}}{\mathrm{d} t}=k_{3} C_{\mathrm{D}} C_{\mathrm{A}}-k_{4} C_{\mathrm{M}} C_{\mathrm{B}}-k_{5} C_{\mathrm{M}} C_{\mathrm{A}}+k_{6} C_{\mathrm{G}} C_{\mathrm{B}} \\
& \frac{1}{m_{\text {cat }}} \frac{\mathrm{d} C_{\mathrm{B}}}{\mathrm{d} t}=k_{1} C_{\mathrm{T}} C_{\mathrm{A}}-k_{2} C_{\mathrm{D}} C_{\mathrm{B}}+k_{3} C_{\mathrm{D}} C_{\mathrm{A}}-k_{4} C_{\mathrm{M}} C_{\mathrm{B}}+ \\
& k_{5} C_{\mathrm{M}} C_{\mathrm{A}}-k_{6} C_{\mathrm{G}} C_{\mathrm{B}}+k_{7} C_{\mathrm{T}} C_{\mathrm{A}}^{3}-k_{8} C_{\mathrm{G}} C_{\mathrm{B}}^{3} \\
& \frac{1}{m_{\text {cat }}} \frac{\mathrm{d} C_{\mathrm{G}}}{\mathrm{d} t}=k_{5} C_{\mathrm{M}} C_{\mathrm{A}}-k_{6} C_{\mathrm{G}} C_{\mathrm{B}}+k_{7} C_{\mathrm{T}} C_{\mathrm{A}}^{3}-k_{8} C_{\mathrm{G}} C_{\mathrm{B}}^{3} \\
& \frac{\mathrm{d} C_{\mathrm{G}}}{\mathrm{d} t}=-\frac{\mathrm{d} C_{\mathrm{B}}}{\mathrm{d} t}
\end{aligned}
$$

Onde, $C$ é a concentração em $\mathrm{g} / \mathrm{cm}^{3}$, $t$ é o tempo em min, $k$ são os parâmetros do modelo cinético, $m_{\text {cat }}$ é a massa de catalisador em g, T é o subscrito para triglicerídeos, D é o subscrito para diglicerídeos, M é o subscrito para monoglicerídeos, B é o subscrito para biodiesel, G é o subscrito para glicerol e A é o subscrito para o álcool (metanol).

Para a obtenção dos k's foi usado o método conhecido como Algoritmo Genético (AG) ao invés de algoritmos clássicos (como Levenberg-Marquardt), pois o problema possui muitos mínimos locais e soluções sem significado físico, o que poderia levar a falhas de convergência caso outro algoritmo fosse empregado. $\mathrm{O}$ AG é um algoritmo inspirado no processo de evolução biológica por meio de seleção natural, onde as potenciais soluções do problema matemático são avaliadas em cada iteração (chamada de geração) e as soluções em potencial das próximas gerações são combinações (ou cruzamentos) das iterações anteriores. As melhores soluções têm maior chance de transmitir suas informações (código genético) para as soluções da geração seguinte e o processo se repete até ocorrer a convergência do problema.

Ao avaliar as soluções em potencial, o AG precisa simular o modelo matemático por meio de um método numérico adequado. Dado que as equações formam um sistema rígido, ou seja, as derivadas apresentam diferentes magnitudes, existe uma dificuldade natural na solução numérica. Para contornar esse problema foi utilizado um método numérico específico para esses tipos de EDOs baseado no método de Rosenbrock de ordem 2, implementado através do comando ode23s do Matlab. Como condições iniciais do problema, foram consideradas a existência de apenas metanol e triglicerídeos no meio reacional a uma razão molar de 6:1.

A qualidade do ajuste das curvas foi avaliada por meio de mínimos quadrados, conforme Equação 7, 
onde $n$ é o número de amostras. A Figura 3 mostra o esquema global da estratégia de ajuste das curvas.

$$
s=\sum_{i=1}^{n}\left(y_{i, \text { modelo }}-y_{i, \text { experimental }}\right)^{2}
$$

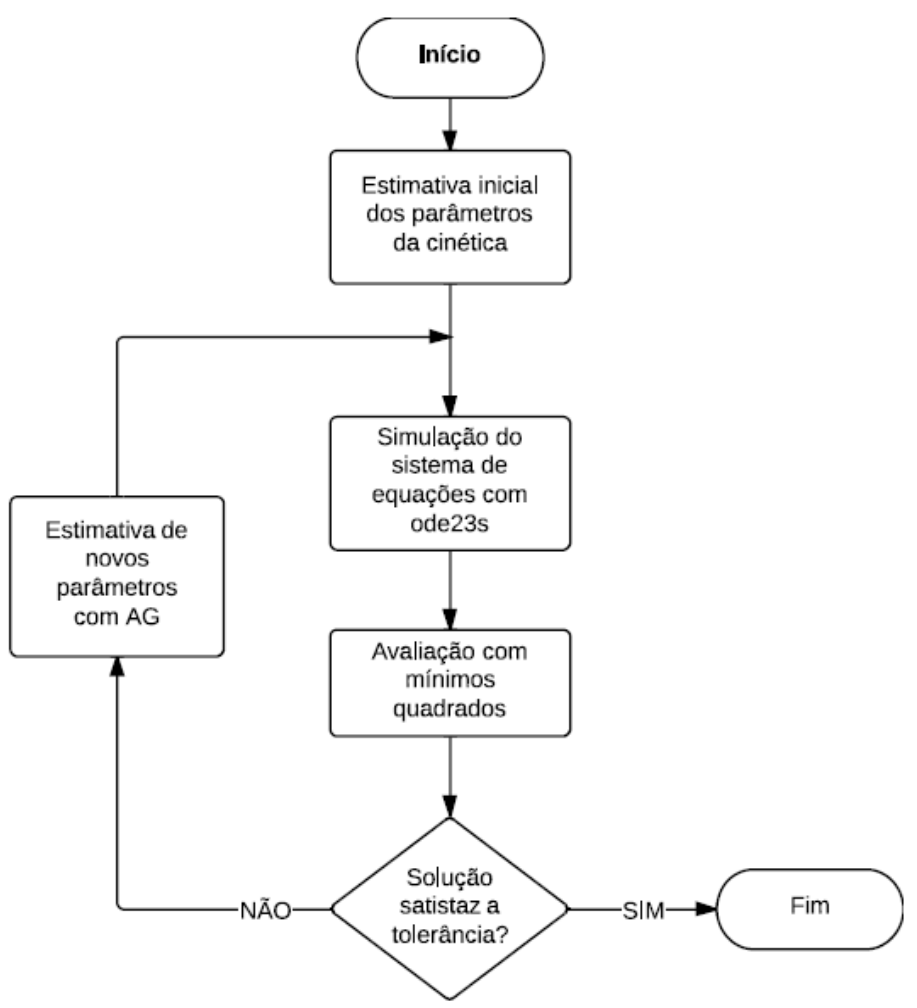

Figura 3 - Fluxograma da estratégia de ajuste do modelo aos dados experimentais.

\section{RESULTADOS E DISCUSSÕES}

A obtenção da peneira molecular MCM-41 pode ser confirmada pelo difratograma de raios X, Figura 4a, onde pode-se perceber a presença dos picos característicos da MCM-41. Esta análise indica que a rota de síntese empregando o trissilicato de sódio como fonte de sílica foi efetiva na obtenção da MCM-41, o método de Mokhonoana et al (2010) levou à formação de um material bem organizado com nítidos picos de difração.

No termograma (Figura 4b) podem ser vistos os 4 picos característicos da perda de massa da CTAMCM-41. O $1^{\circ}$ pico se refere à água fisicamente adsorvida, o $2^{\circ}$ está associado à decomposição do surfactante, o $3^{\circ}$ pico está relacionado com a combustão de espécies restantes de carbono e o $4^{\circ}$ pico se deve a desidroxilação (Martins e Cardoso, 2007) totalizando uma perda de massa de cerca de 59\%. Assim é possível comprovar que os cátions orgânicos $\left(\mathrm{CTA}^{+}\right)$pertencem a estrutura, região 2. 


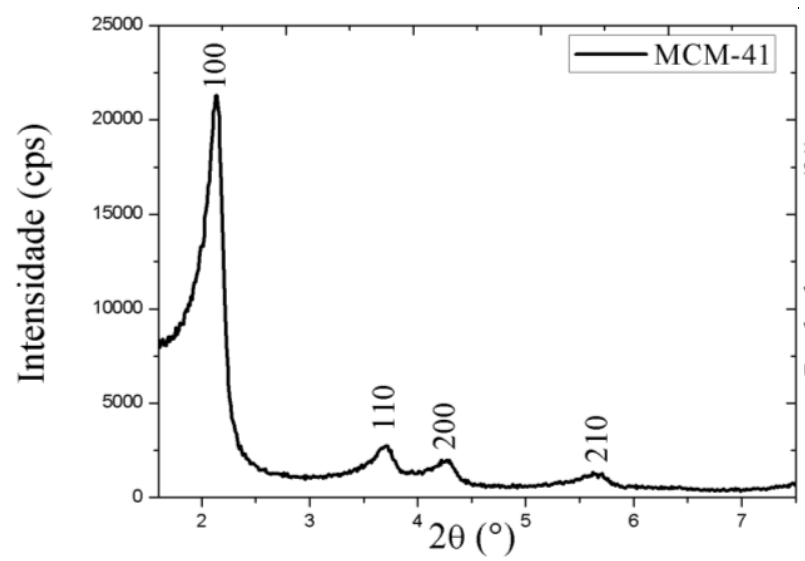

(a)

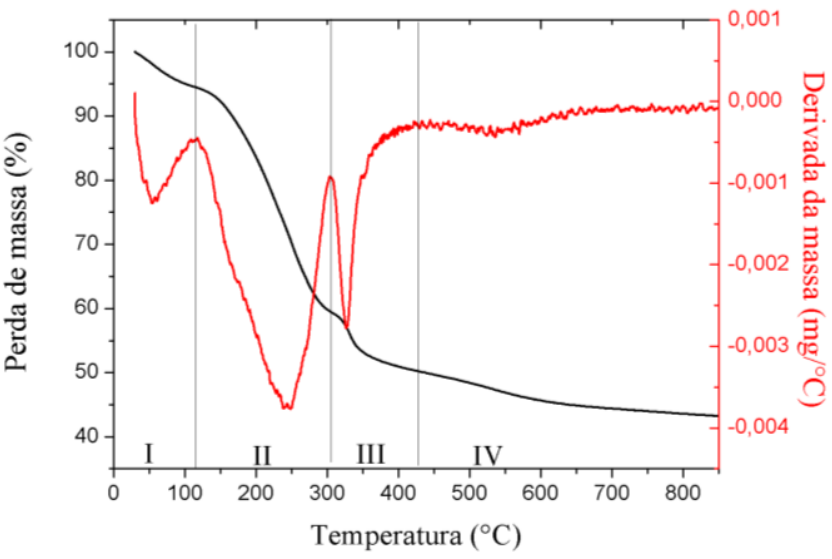

(b)

Figura 4 - Caracterização do catalisador: (a) difratograma de raios X e (b) termograma.

Quanto aos dados obtidos via cromatografia líquida (HPLC), a técnica identificou apenas a composição dos óleos (biodiesel, monoglicerídeos, diglicerídeos e triglicerídeos) em função de sua calibração. Assim, a qualidade do ajuste (Equação 7) levou em consideração apenas a composição mássica obtida para estes componentes.

O resultado do ajuste do modelo matemático aos dados experimentais está apresentado na Figura 5. É possível observar que em geral o modelo conseguiu aproximar o comportamento da reação com razoável precisão como indicado pelo coeficiente $\mathrm{R}^{2}$ para o biodiesel $(0,975)$, monoglicerídeos $(0,993)$, diglicerídeos $(0,975)$ e triglicerídeos $(0,980)$. Os valores dos parâmetros cinéticos estão apresentados na Tabela 1.

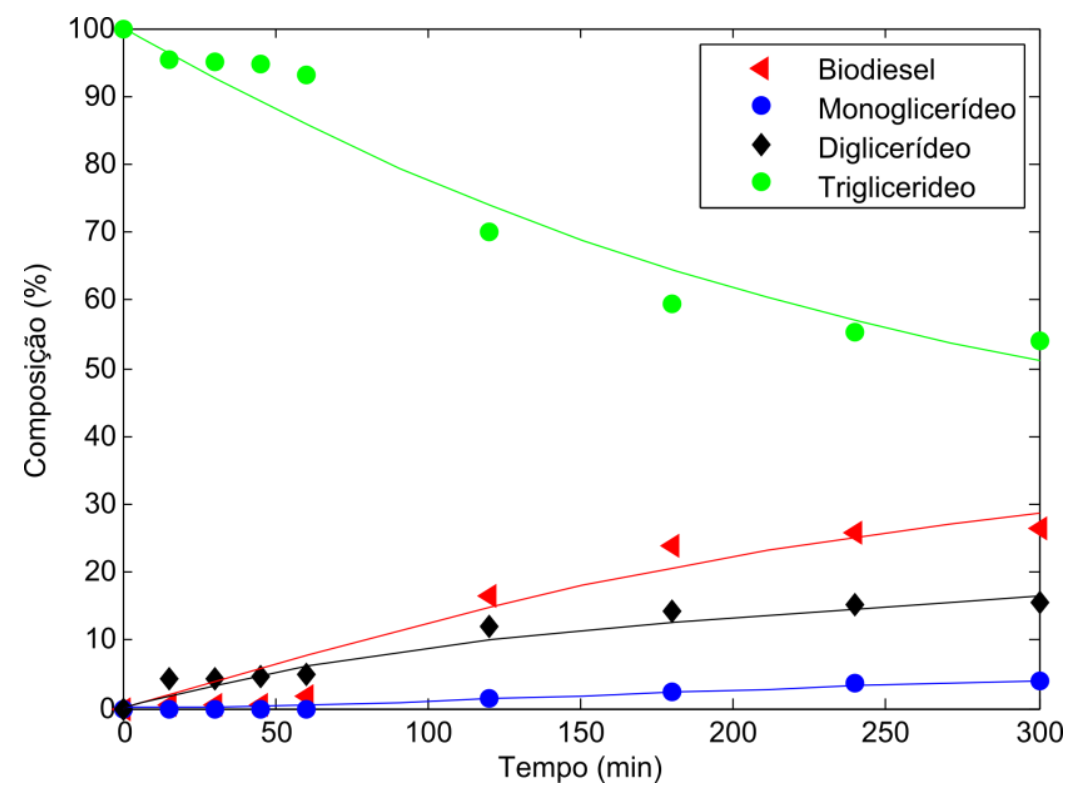

Figura 5 - Ajuste do modelo aos dados experimentais. 
Tabela 1 - Parâmetros obtidos para o modelo.

\begin{tabular}{|c|c|}
\hline Parâmetro & Valor \\
\hline$k_{1}$ & $3,379 \cdot 10^{-2}$ \\
\hline$k_{2}$ & $3,005 \cdot 10^{-1}$ \\
\hline$k_{3}$ & $9,312 \cdot 10^{-2}$ \\
\hline$k_{4}$ & 4,789 \\
\hline$k_{5}$ & $4,444 \cdot 10^{-1}$ \\
\hline$k_{6}$ & 17,62 \\
\hline$k_{7}$ & $3,766 \cdot 10^{-5}$ \\
\hline$k_{8}$ & 7,706 \\
\hline
\end{tabular}

Os primeiros pontos referentes as composições de triglicerídeo e diglicerídeo apresentaram um comportamento diferente do esperado para esse sistema reacional, pois nos dois primeiros minutos a reação converte aproximadamente 5\% do triglicerídeo para em seguida desacelerar por aproximadamente 8 min, para depois continuar normalmente. Ainda não é possível explicar fisicamente esse fenômeno, que é reprodutível nas condições operacionais apresentadas, mas o modelo é capaz de aproximar o comportamento nessa faixa.

Por fim, visto que apenas dados para a temperatura de $80^{\circ} \mathrm{C}$ foram utilizados, o modelo é válido apenas nas proximidades dessa temperatura, mas pode ser generalizado para outros valores de massa de catalisador.

\section{CONCLUSÕES}

Neste trabalho foi apresentada a síntese da MCM-41 baseada no procedimento de Mokhonoana et al (2010) e um modelo matemático para sua aplicação na reação de transesterificação de óleo de soja. Através de difratogramas de raios X foi possível comprovar a obtenção da MCM-41 e por meio de termogravimetria, mostrou-se a existência da $\mathrm{CTA}^{+}$no material. $\mathrm{O}$ modelo matemático ajustou-se bem aos dados experimentais, entretanto, sua aplicação está restrita às proximidades da temperatura de $80^{\circ} \mathrm{C}$.

\section{REFERÊNCIAS}

Os autores gostariam de agradecer ao apoio financeiro da PROCAD/CAPES e CNPq e a professora Simoni Meneghetti pela colaboração na execução do trabalho.

\section{REFERÊNCIAS}

BORGES, M. E.; DÍAZ, L. Recent developments on heterogeneous catalysts for biodiesel production by oil esterification and transesterification reactions: a review. Renewable and Sustainable Energy Reviews, n. 16, p. 2839-2849, 2012.

COSTA, P. P. K. G. Catalisadores químicos utilizados na síntese de biodiesel. $1^{\mathrm{a}}$. ed. Brasília: Embrapa, 2011. 
FABIANO, D. P. Síntese e avaliação de sílica tipo M41S contendo cátions CTA em transesterificação catalítica. 137 p. Tese (Mestrado), Universidade Federal de São Carlos, São Carlos, 2010.

FABIANO, D. P.; HAMAD, B.; CARDOSO, D.; ESSAYEM, N. On the understanding of the remarkable activity of template-containing mesoporous molecular sieves in the transesterication of rapeseed oil with ethanol. Journal of Catalysis, n. 276, p. 190-196, 2010.

KUBOTA, Y.; NISHIZAKI, Y.; IKEYA, H.; SAEKI, M.; HIDA, T.; KAWAZU, S.; YOSHIDA, M.; FUJI, H.; SUGI, Y. Organic-silicate hybrid catalysts based on various defined structures for knoevenagel condensation. Microporous and Mesoporous Materials, n. 70, p. 135-149, 2004.

MARTINS, L.; CARDOSO, D. Aplicação catalítica de peneiras moleculares básicas micro e mesoporosas. Química Nova, v. 29, n. 2, p. 358-364, 2006.

MARTINS, L.; CARDOSO, D. Influence of surfactant chain length on basic catalytic properties of SiMCM-41. Microporous and Mesoporous Materials, v. 106, p. 8-16, 2007.

MOKHONOANA, M. P.; COVILLE, N. J. Synthesis of [Si]-MCM-41 from teos and water glass: the water glass-enhanced condensation of teos under alkaline conditions. J. Sol-Gel Sci. Technol., n. 54, p. 83-92, 2010.

NOUREDDINI, H.; ZHU, D. Kinetics of transesterification of soybean oil. Biocatalysis Articles, $\mathrm{n}$. 74, p. $1457-1463,1997$.

SEMWAL, S.; ARORA, A. K.; BADONI, R. P.; TULI, D. K. Biodiesel production using heterogeneous catalysts. Bioresource Tecnology, n. 102, p. 2151-2161, 2011. 